\title{
REPORT OF THE TIRI WORKSHOP, SATURDAY 13 AUGUST 1994
}

\author{
STEINAR GULLIKSEN
}

Radiological Dating Laboratory, Norwegian Institute of Technology, N-7034 Trondheim, Norway

and

MARIAN SCOTT

Department of Statistics, University of Glasgow, University Gardens, Glasgow, G12 8QW, Scotland

The results from the Third International Radiocarbon Intercomparison (TIRI) were presented and discussed at the Workshop. This was the first opportunity for many of the participants to see all of the results and to be able to discuss them. More than 60 people representing both consumers and providers of dates participated in the Workshop.

The first session was taken by the organizers of TIRI (Scott, Harkness, Miller, Cook and Begg) and comprised an overview of the project, the samples used, their treatment and, finally, a preliminary (mainly graphical) analysis of the results. One of the main functions of TIRI is to provide an independent and objective assessment of analytical quality, taking the form of a "regular medical checkup". As such, a suite of 13 samples was prepared, all of them natural and spanning activities from modern to background. Some of the samples had been pretreated and homogenized before dispatch (e.g., humic acid), whereas others were simply packaged (e.g., tree-ring samples). In the first instance, TIRI is testing all stages of a laboratory's procedures, and in the first analysis of the results, the emphasis is on understanding any differences in the actual measurements. It is clear that in making use of "known-age" material such as tree rings, a further analysis of such results is also of particular interest to users. Samples were designated "core" or "optional", all participating laboratories received the 6 core samples, and the remaining 7 optional samples were available at a laboratory's discretion. A summary of all the results for each sample was presented, outlying or extreme values were identified and preliminary consensus values for the sample activities evaluated. A discussion followed on the topics of sample homogeneity and on the question of differing measured ${ }^{14} \mathrm{C}$ contents depending on the chemical fraction dated. In addition, several AMS labs raised the question of the selection of small samples from the large sample provided through TIRI for AMS dating. The preliminary results also brought to light some interesting findings concerning the two "near-background" samples included in TIRI (not least the difficulty of carrying out any statistical analysis given the different ways of quoting the results). The difficulties that these types of samples present were re-emphasized in a later presentation by Alan Hogg on a Kauri reference material.

The afternoon session continued with presentations by TIRI participants. It was of particular interest to see how individual laboratories were able to use the TIRI results to identify problems in their operation and to correct them. The final session dealt with related and future work, with, as mentioned earlier, a presentation by Alan Hogg on a new IAEA ${ }^{14} \mathrm{C}$ quality assurance material and a suggestion by Lloyd Currie regarding a need to consider a small-sample intercomparison. Finally, the organizers of TIRI indicated a willingness to continue their work and to undertake to organize the next intercomparison along similar lines to TIRI, but with increased emphasis on small, old samples. To this end, they would begin formulating a protocol and generally seek information and views on appropriate samples for the next intercomparison. It was clear that including "known-age" material was seen as important, and thus, tree-ring samples will again be sought. The next TIRI may include a peat sample from between two known-age tephra layers. The importance of sample homogeneity was stressed; the organizers will encourage the participation of AMS labs in the sample testing. 


\section{Preliminary Consensus Values}

Consensus values for each sample were evaluated using the same method used in the characterization of the IAEA reference samples (Rozanski et al. 1992). Briefly, a preliminary, robust consensus value (rcv) was evaluated (the median of all the results with identified outliers removed) for each of the samples. To evaluate the final consensus value, the standardized difference (sd) between the rcv and each result is calculated (sd=(result-rcv)/quoted uncertainty). If the sd exceeds 2 , then that result is not used in any subsequent calculation. In this way, results that do not lie within \pm 2 quoted uncertainties of the rcv are removed. The final rcv is calculated as a weighted average of the remaining results. Tables $1 \mathrm{~A}$ and $1 \mathrm{~B}$ show the rcvs for the Stages 1 and 2 samples, evaluated using the criterion stated above.

Table 1A. Preliminary Consensus Values for Stage 1 Samples

\begin{tabular}{lcc} 
Sample & $\begin{array}{c}\text { Consensus } \\
\text { value }\end{array}$ & $\begin{array}{c}\text { Estimated } \\
\text { precision }(1 \text { o })\end{array}$ \\
\hline A: Barley mash & $116.35 \mathrm{pMC}$ & 0.0084 \\
B: Belfast pine & 4503 & 6 \\
C: IAEA cellulose & $129.7 \mathrm{pMC}$ & 0.08 \\
D: Hekla peat & 3810 & 7 \\
E: Ellanmore humic & 11,129 & 12 \\
F: Icelandic doublespar & 46,750 & 208 \\
& $0.18 \mathrm{pMC}$ & 0.006 \\
\hline
\end{tabular}

Table 1B. Preliminary Consensus Values for Stage 2 Samples

\begin{tabular}{lcc}
\hline Sample & $\begin{array}{c}\text { Consensus } \\
\text { value }\end{array}$ & $\begin{array}{c}\text { Estimated } \\
\text { precision }(1 \sigma)\end{array}$ \\
\hline G: Fuglaness wood & 39,784 & 620 \\
H: Ellanmore whole peat & 11,152 & 23 \\
I: Travertine & 11,060 & 17 \\
J: Crannog wood & 1605 & 8 \\
K: Turbidite carbonate & 18,155 & 34 \\
L: Whale bone & 12,788 & 30 \\
M: Icelandic peat & 1682 & 15 \\
\hline
\end{tabular}

\section{REFERENCE}

Rozanski, K., Stichler, W., Gonfiantini, R., Scott, E. M., Beukens, R. P., Kromer, B. and van der Plicht, J. 1992 The IAEA

${ }^{14} \mathrm{C}$ intercomparison exercise 1990 . In Long, A. and Kra, R. S., eds., Proceedings of the 14 th International ${ }^{14} \mathrm{C}$ Conference.

Radiocarbon 34(3): 506-519. 